\title{
The effect of TEAS on the quality of early recovery in patients undergoing gynecological laparoscopic surgery: a prospective, randomized, placebo- controlled trial
}

\author{
Xiangdi Yu*, Fangxiang Zhang and Bingning Chen
}

\begin{abstract}
Introduction: In current study we assessed the effect of transcutaneous electrical acupoint stimulation (TEAS) on the quality of early recovery in patients undergoing gynecological laparoscopic surgery.

Methods: Sixty patients undergoing gynecological laparoscopic surgery were randomly assigned to TEAS (TEAS group) or control group (Con group). TEAS consisted of $30 \mathrm{~min}$ of stimulation $(12-15 \mathrm{~mA}, 2 / 100 \mathrm{~Hz}$ ) at the acupoints of Baihui (GV20), Yingtang (EX-HN-3), Zusanli (ST36) and Neiguan (PC6) before anesthesia. The patients in the Con group had the electrodes applied, but received no stimulation. Quality of recovery was assessed using a 40-item questionnaire as a measure of quality of recovery (QoR-40; maximum score 200) scoring system performed on preoperative day 1 (T0), postoperative day 1 (T1) and postoperative day 2 (T2); 100-mm visual analogue scale (VAS) scores at rest, mini-mental state examination (MMSE) scores, the incidence of nausea and vomiting, postoperative pain medications, and antiemetics were also recorded. Results: QoR-40 and MMSE scores of T0 showed no difference between two groups (QoR-40: $197.50 \pm 2.57$ vs. $195.83 \pm 5.17$ ), (MMSE: $26.83 \pm 2.74$ vs. $27.53 \pm$ 2.88). Compared with the Con group, QoR-40 and MMSE scores of T1 and T2 were higher in the TEAS group $(P<$ 0.05) (QoR-40: T1, $166.07 \pm 8.44$ vs. $175.33 \pm 9.66 ;$ T2, $187.73 \pm 5.47$ vs. $191.40 \pm 5.74$ ), (MMSE: T1, $24.60 \pm 2.35$ vs. $26.10 \pm 2.78 ; T 2,26.53 \pm 2.94$ vs. $27.83 \pm 2.73)$. VAS scores of T1 and T2 were lower $(P<0.05)$ in the TEAS group (T1, $4.73 \pm 1.53$ vs. $3.70 \pm 1.41 ; T 2,2.30 \pm 0.95$ vs. $1.83 \pm 0.88$ ); the incidence of postoperative nausea and vomiting (PONV), remedial antiemetics and remedial analgesia was lower in the TEAS group ( $P<0.05)$ (PONV: $56.7 \%$ vs. 23.3\%; incidence of remedial antiemetics: $53.3 \%$ vs. $23.3 \%$; incidence of remedial analgesia: $80 \%$ vs. $43.3 \%$ ).

Conclusion: The use of TEAS significantly promoted the quality of early recovery, improved MMSE scores and reduced the incidence of pain, nausea and vomiting in patients undergoing gynecological laparoscopic surgery.

Trial registration: ClinicalTrials.gov, NCT02619578. Registered on 2 December 2015.

Trial registry name: https://clinicaltrials.gov
\end{abstract}

Keywords: Transcutaneous electric acupoint stimulation (TEAS), Gynecological laparoscopic surgery, QoR-40, MMSE, VAS

\footnotetext{
* Correspondence: Xiangdi_Yu@163.com

Department of Anesthesiology, Guizhou Provincial People's Hospital, No. 83

Zhongshan Road Nanming district, Guiyang City, Guizhou Province, China
}

(c) The Author(s). 2020 Open Access This article is distributed under the terms of the Creative Commons Attribution 4.0 International License (http://creativecommons.org/licenses/by/4.0/), which permits unrestricted use, distribution, and reproduction in any medium, provided you give appropriate credit to the original author(s) and the source, provide a link to the Creative Commons license, and indicate if changes were made. The Creative Commons Public Domain Dedication waiver (http://creativecommons.org/publicdomain/zero/1.0/) applies to the data made available in this article, unless otherwise stated. 


\section{Introduction}

During the past four decades, gynecologic laparoscopy has evolved from a limited method to an advanced operative approach that frequently serves as a substitute for laparotomy. The advantages of laparoscopy over laparotomy include less postoperative pain, shorter hospital stays, and reduced blood loss [1-3]. However, during surgery $\mathrm{CO}_{2}$ increases the intra-abdominal and intrathoracic pressure, which leads to cardiac output decrease and increase in sympathetic activity in a reflex. On the other hand, $\mathrm{CO}_{2}$ accumulation in the body leads to hypercapnia, which indirectly stimulates aortic body chemosensory organs and carotid sinus, increasing the concentration of plasma catecholamines, cortisol and vasopressin [4, 5], these responses have an important impact on patient recovery after surgery.

Acupuncture is an ancient Chinese method to treat diseases and relieve pain. Transcutaneous electrical acupoint stimulation (TEAS), a noninvasive adjunctive intervention based on acupuncture, has been widely accepted and used worldwide [6]. To date, multiple studies have demonstrated that TEAS could reduce the use of intra-operative opioid drugs and the incidence of postoperative nausea and vomiting (PONV), and improve postoperative cognitive function [7, 8]. However, whether TEAS could improve the quality of early recovery after gynecologic laparoscopy is unknown. In this study we therefore investigated the effects of TEAS at the acupoints of Baihui (GV20), Yingtang (EX-HN3), Zusanli (ST36) and Neiguan (PC6) on the quality of early recovery in patients undergoing gynecological laparoscopic surgery.

\section{Methods}

This is a double-blind randomized controlled trial (RCT). The study was conducted in accordance with the Declaration of Helsinki and was approved by the local Clinical Research Ethics Committee. Written informed consent was obtained from each participant. The study was approved by the Ethics Committee of Guizhou Provincial People's Hospital.

\section{Patient population}

Sixty patients undergoing elective gynecological laparoscopic surgery at Guizhou province people's hospital with an American Society of Anesthiologists (ASA) classification physical status of I-II were recruited between November 2013 and November 2014; written consent was obtained from all patients. Exclusion criteria were recent use of TEAS or acupuncture, neural damage or infection along the meridian at which the acupoints lay, use of antiemetic in the previous week, regular use of opioids, hepatic dysfunction, confirmed renal impairment, diabetes mellitus, cognitive dysfunction and conversion to laparotomy during gynecologic laparoscopy.

\section{Randomization and blinding}

Patients were assigned by the nurse to either TEAS stimulus (TEAS group) or control (Con group) on the basis of random numbers generated by a computer before the start of surgery (Fig. 1). Only the acupuncturist was informed by the nurse of the randomization allocation, just before the onset of TEAS. None of the anesthesiologists, surgeons, physicians in the post-anesthesia care unit (PACU), or participants were aware of the allocation. Blinding of the patients was ensured by using gel electrodes in the same therapeutic setting, which has previously been proved to be a successful strategy [9].

\section{TEAS protocol}

An experienced acupuncturist performed TEAS for $30 \mathrm{~min}$ before anesthesia. According to the theory of traditional Chinese medicine, bilateral Baihui (GV20), Yingtang (EX-HN3), Zusanli (ST36) and Neiguan (PC6) were chosen as the acupuncture points. These acupoints were identified according to traditional anatomic localization (Fig. 2). Gel electrodes were applied to the skin after it had been cleaned with ethyl alcohol. The acupoints were then stimulated electrically with an intensity of $12-15 \mathrm{~mA}$ and dense-disperse frequency of $2 / 100 \mathrm{~Hz}$ for $30 \mathrm{~min}$, using the Hwato electronic acupuncture treatment instrument (model SDZ-V, Suzhou Medical Appliances Co., Ltd., Suzhou, China). The intensity was adjusted to maintain slight twitching of local muscles according to individual maximum tolerance, indicating a satisfactory De-Qi phenomenon and thus adequate stimulation. The patients in the control group had the electrodes applied but received no stimulation.

\section{Anesthesia and perioperative management}

One surgeon conducted all surgery according to a standard protocol; surgery commenced between 8:30 and 1:00 p.m. Anesthesia was induced intravenously (i.v.) with propofol and remifentanil using a targetcontrolled infusion (TCI) system. After loss of consciousness, vecuronium $\left(0.1 \mathrm{mg} \cdot \mathrm{kg}^{-1}\right)$ was administered i.v., and patients were orotracheally intubated 5 min later. Anesthesia was maintained with TCI of propofol and remifentanil. The depth of anesthesia was monitored using the bispectral index (BIS). Concentrations at the affected sites of propofol and remifentanil were adjusted to the hemodynamic index and BIS. Patients' lungs were mechanically ventilated in a volume-controlled mode with a tidal volume of 8 $\mathrm{ml} \cdot \mathrm{kg}^{-1}$ body weight during the operation. In both groups, remifentanil and propofol infusions were stopped $5 \mathrm{~min}$ before the end of surgery. Patients were extubated and transferred to the Post Anasthesia Care Unit (PACU) after surgery. 


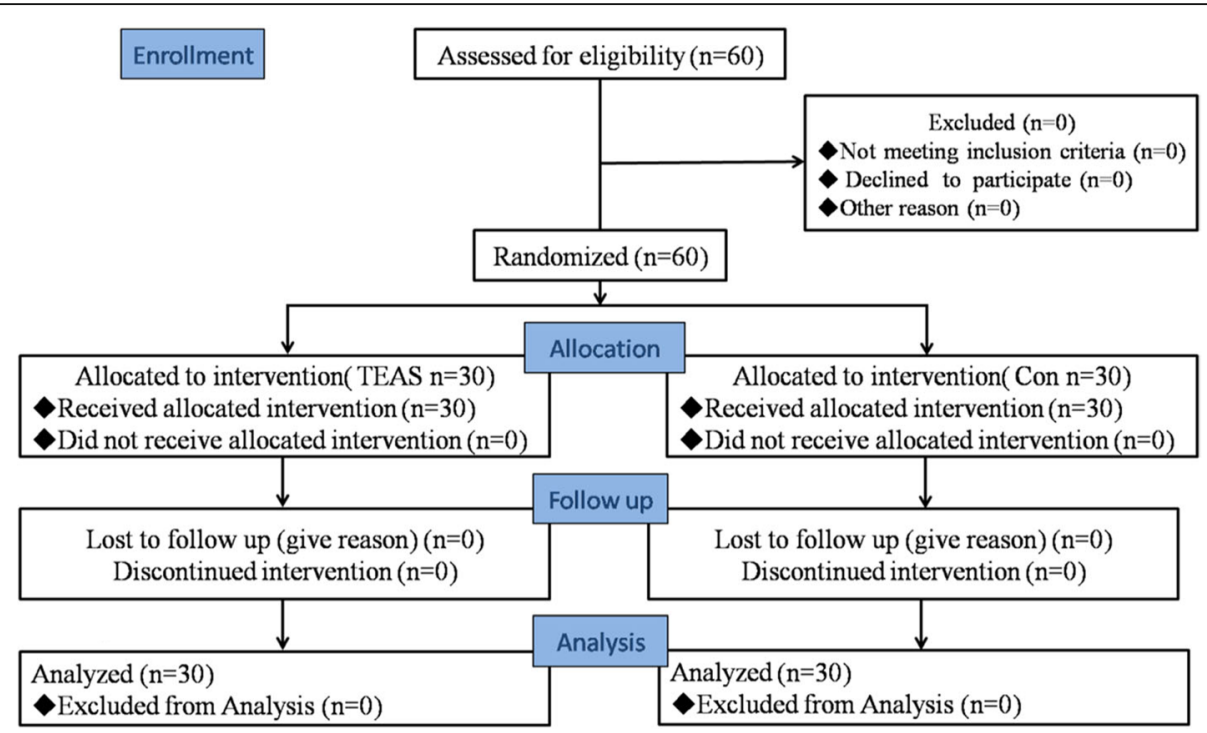

Fig. 1 Consolidated standards of reporting trials (Consort) flowchart. TEAS, Transcutaneous electrical acupoint stimulation

\section{End points}

The primary end points were postoperative quality of recovery and cognitive functioning, and the secondary endpoint was anesthesia-related side effects, including pain scores, the incidence of nausea and vomiting and use of postoperative pain medications and antiemetics.

The Quality of Recovery-40 (QoR-40) is a validated scale with five domains [10-12]. These measure physical comfort, emotional state, physical independence, psychological support and pain. Each domain is scored to a maximum global score of 200. QoR-40 scores have been found to be associated with quality-of-life scales, patient satisfaction indices [13] and postoperative pain [14]. In the present study, QoR-40 evaluation was performed on preoperative day 1 (T0), postoperative day 1 (T1) and postoperative day 2 (T2).

The Mini-Mental State Examination (MMSE) is one of the most widely used assessment instruments of postoperative cognitive functioning, and screens domains of orientation to time and place, attention and memory, concentration, language and praxis [15]. Patient cognitive function was assessed using the MMSE on days T0, $\mathrm{T} 1$ and $\mathrm{T} 2$.

The visual analog scale (VAS) is widely used in behavioral science, and previous studies have reported their usefulness and validity $[16,17]$. We used VAS scores to determine participants' levels of pain at rest on postoperative day 1 (T1) and postoperative day 2 (T2).

\section{Data collection}

Baseline data included demographics, body mass index (BMI) and ASA physical status. Surgical information recorded included anesthesia duration, surgery duration, estimated blood loss and all other intraoperative medications. BN, who was blinded to grouping, conducted the data collection and administered the questionnaires; FZ analyzed the data.

\section{Sample size}

Sample size calculation was based on the primary outcome of QoR-40 scores and MMSE. We calculated that 16 patients in each group were required to detect a $30 \%$ difference between the groups, assuming two-sided type I error $(\alpha)$ of 0.05 and power of $80 \%$. To account for potential loss to follow up and enable greater statistical power for secondary analysis, the sample size was increased to 60 patients (30 per group).

\section{Statistical analysis}

All statistical analysis was based on the intention-totreat principle. Normally distributed numeric data were analyzed using the $t$ test and are reported as mean and standard deviation (SD). Variables measured at multiple time points were analyzed using repeated-measures analysis of variance. Dichotomous variables were presented as the number of patients (percent) and analyzed using the chi-square $\left(X^{2}\right)$ test and are reported as counts with percentages. Statistical significance was assessed at $P<$ 0.05 .

\section{Results}

\section{Patient characteristics}

Complete datasets were collected for all 60 participants and all the data were analyzed (Fig. 3). The characteristics of patients such as age, height, BMI, ASA physical status, anesthesia duration, surgery duration, estimated 


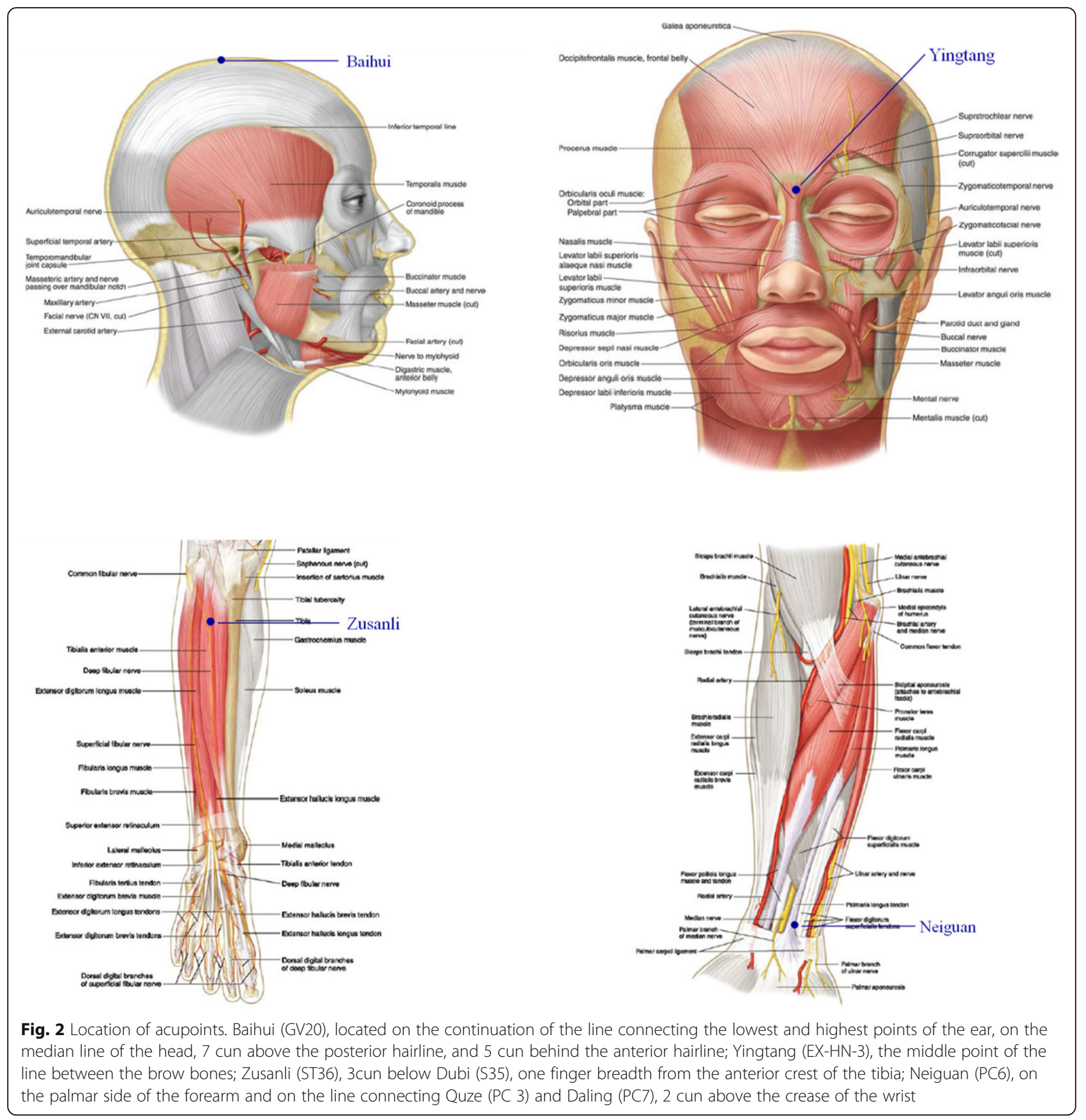

blood loss and fluid balance did not differ between the groups (Table 1).

\section{Scores on the QoR-40 and MMSE (primary outcomes)}

When evaluating the QoR-40, the global scores at baseline (T0) were similar. Following the procedure, global scores at $\mathrm{T} 1$ and $\mathrm{T} 2$ were significantly higher in the TEAS group $(P=0.040$ and $P=0.015$ for global QoR- 40 at T1 and T2, respectively); when T0 was used as a covariate for a repeated measures model of $\mathrm{T} 1$ and $\mathrm{T} 2$, the significances for the interaction of group and time course were 0.003 and 0.036 , respectively, indicating better quality of recovery with TEAS treatment (Fig. 4).

There were no significant differences in the baseline (T0) MMSE score for cognitive functioning in the two groups; however, the scores were significantly lower in the TEAS group compared with the Con group $(P=$ 0.048 and $P=0.02$ for $\mathrm{T} 1$ and $\mathrm{T} 2$, respectively); when T0 was used as a covariate for a repeated measures model of $\mathrm{T} 1$ and $\mathrm{T} 2$, the significances for the interaction of group and time course were 0.007 and 0.046 , respectively (Fig. 5). 


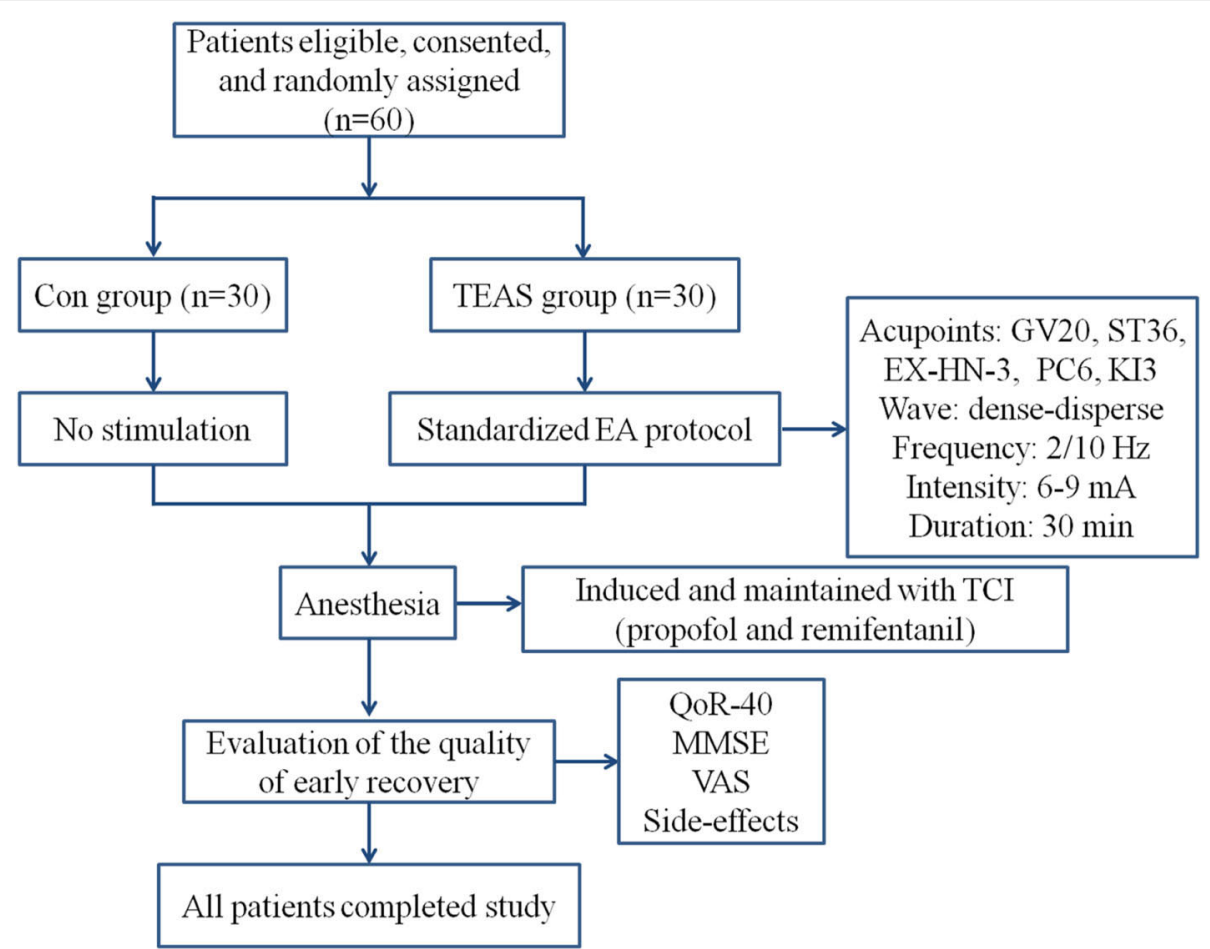

Fig. 3 Flow diagram showing the study procedures and number of patients. TEAS, transcutaneous electric acupoint stimulation; TCI, target controlled infusion; EA, electroacupuncture; Con, control; MMSE, Mini Mental State Examination; VAS, visual analogue scale

Pain assessment and the incidence of nausea and vomiting, postoperative pain medications and antiemetics (secondary outcomes)

The VAS scores for pain at rest were significantly lower in the TEAS group compared with the Con group ( $P=$ 0.042 and $P=0.26$ for T1 and T2, respectively) (Table 2). The frequency of nausea and vomiting within the first 24h after surgery is shown in Table 3: 7 patients in the TEAS group and 17 patients in the Con group complained of nausea or vomiting with the first $24 \mathrm{~h}$ after

Table 1 Patient characteristic and clinical characteristics of participants

\begin{tabular}{lll}
\hline & $\begin{array}{l}\text { Contol group } \\
(n=30)\end{array}$ & $\begin{array}{l}\text { TEAS group } \\
(n=30)\end{array}$ \\
\hline Age, years & $45.9(17.5)$ & $48.5(16.2)$ \\
Height (cm) & $157.9(5.2)$ & $158.3(6.7)$ \\
Body mass index & $22.1(4.5)$ & $22.0(3.4)$ \\
ASA class & & \\
$\quad$ I & 23 & 22 \\
$\quad$ || & 7 & 8 \\
Anesthesia duration (min) & $190.3(65.0)$ & $224.2(88.0)$ \\
Operation duration (min) & $125.5(62.1)$ & $150.6(76.4)$ \\
Intra-operative fluids (ml) & $1710.6(533.6)$ & $1755.8(479.3)$ \\
\hline $\begin{array}{l}\text { Data are presented as mean (SD). TEAS transcutaneous electrical acupoint } \\
\text { stimulation, ASA American Society of Anesthesiologists }\end{array}$
\end{tabular}

surgery; 7 patients in the TEAS group and 16 patients in the Con group required tropisetron as antiemetic rescue therapy; 13 patients in the TEAS group and 24 patients in the Con group required sufentanil as analgesic rescue therapy (Table 3).

\section{Discussion}

In this blinded RCT, use of TEAS at the acupoints of Baihui (GV20), Yingtang (EX-HN3), Zusanli (ST36) and Neiguan (PC6) for 30 min before anesthesia in patients undergoing gynecological laparoscopic surgery improved QoR and MMSE scores, reduced VAS scores and the need for pain medications and resulted in less postoperative nausea, vomiting and need of antiemetics. Our findings demonstrated that TEAS could promote patients' recovery after laparoscopic surgery.

Laparoscopic surgery, with the advantages of less trauma and rapid recovery, is a method that is better accepted by patients. However, $\mathrm{CO}_{2}$ absorption and elevation of intra-abdominal pressure (IAP) from artificial pneumoperitoneum may impact patients' postoperative recovery [18]. Previous studies have reported that the rates of postoperative symptoms in patients undergoing laparoscopic surgery were approximately $45 \%$ for pain, $17 \%$ for nausea, $8 \%$ for vomiting, $17 \%$ for headaches, $42 \%$ for drowsiness, $18 \%$ for dizziness, and $21 \%$ for fatigue respectively [19]. Therefore it is important to 


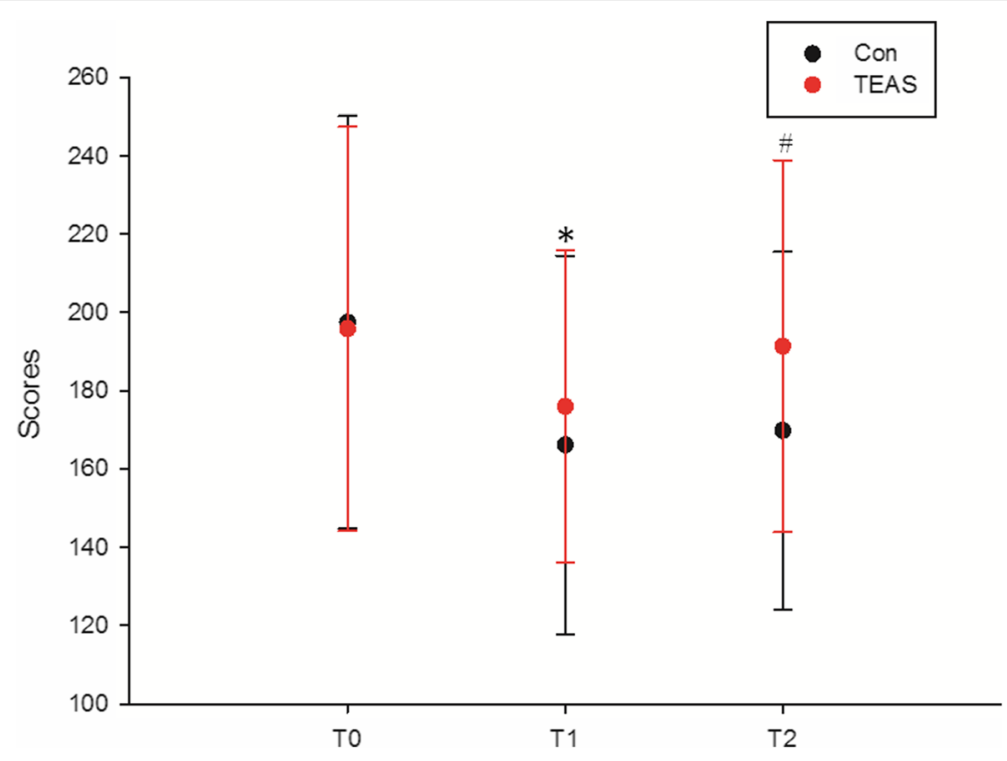

Fig. 4 Scores on the global 40-item questionnaire as a measure of quality of recovery (QoR-40). When T0 was used as a covariate for a repeated measures model of T1 and T2, the significance of the interaction of group and time course was 0.003 and 0.036 , respectively; ${ }^{*} P=0.040$ control (Con) vs. electric acupoint stimulation (TEAS) at postoperative day 1 (T1); \#P=0.015 Con. vs. TEAS at postoperative day 2 (T2)

identify an easy and effective method to improve patient recovery after laparoscopic surgery.

Acupuncture has been used in China to treat a wide variety of diseases for thousands of years. Its major advantages are that it is minimally invasive, safe for highrisk patients and provides effective relief with a low risk of complications [20]. TEAS is the combination of traditional Chinese acupuncture and modern electrical techniques. An increasing number of clinical trials indicate that acupuncture and TEAS may be effective in reducing perioperative analgesic requirements, postoperative pain [21], and PONV [7]. Increasing evidence from animal experiments has shown that TEAS can attenuate cognitive deficits by means of maintaining sympathetic-parasympathetic balance, inhibiting apoptosis [22] and neuroinflammation [23] and regulating

\section{Scores on MMSE}

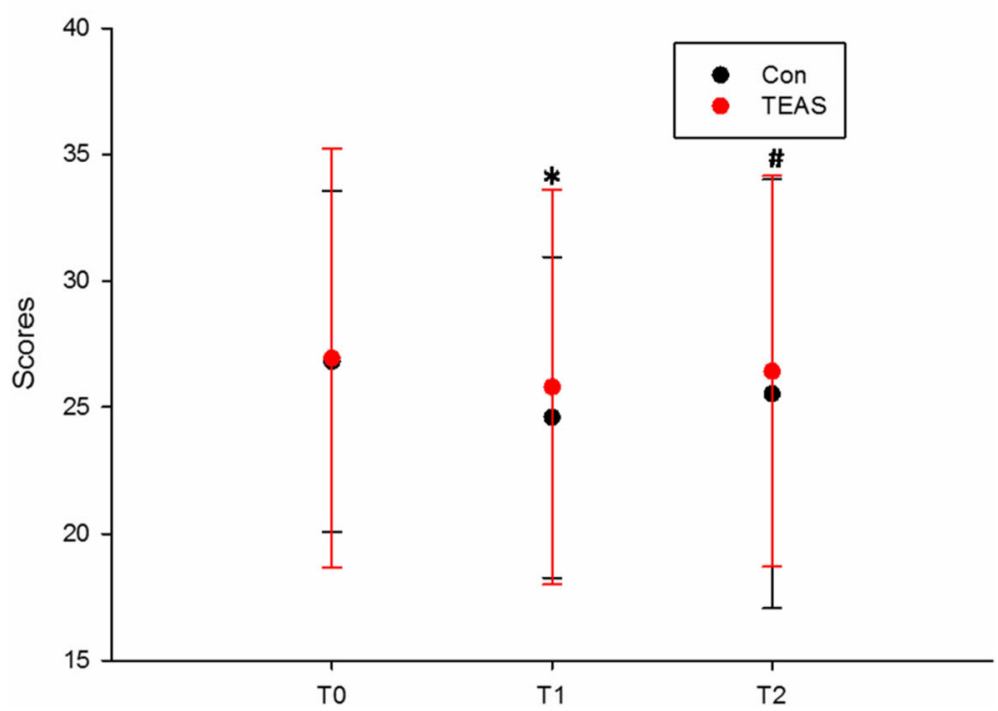

Fig. 5 Scores on the Mini Mental State Examination (MMSE) 
Table 2 Scores on the VAS

\begin{tabular}{lllll}
\hline & Group & T0 & T1 & T2 \\
\hline VAS scores & Con & - & $4.73(1.53)$ & $2.30(0.95)$ \\
& TEAS & - & $3.70(1.53)^{*}$ & $1.83(0.98)^{*}$ \\
\hline
\end{tabular}

Data are presented as mean (SD). VAS visual analogue scale, TO baseline, $T 1$ postoperative day $1, T 2$ postoperative day $2, T E A S$ transcutaneous electrical acupoint stimulation

${ }^{*} P<0.05$ vs. control group

cell proliferation [24] in the hippocampus. Furthermore, TEAS at Zusanli (ST36) has been shown to accelerate the recovery of gastrointestinal motility after colorectal surgery [25].

In the present study, we found that TEAS at Baihui (GV20), Yingtang (EX-HN3), Zusanli (ST36) and Neiguan (PC6) for $30 \mathrm{~min}$ before anesthesia could promote the quality of early recovery, improve cognitive function, ameliorate postoperative pain and reduce the incidence of nausea and vomiting in patients undergoing gynecological laparoscopic surgery. The possible mechanisms were described as follows.

First, evidence from previous studies has shown that acupuncture and electroacupuncture (EA) performed at Baihui (GV20), Zusanli (ST36) and Yingtang (EX-HN-3) could regulate the function of the hypothalamicpituitary-adrenal (HPA) axis [26] and antagonize the hyper-function of the HPA axis [27], possibly by regulating the relative enzymes (protein kinase $\mathrm{A}$ and protein kinase $C$ in the experiment) in the signaling pathway in hippocampal cells. Evidence from animal experiments demonstrates that EA could significantly reduce the content of corticotrophin releasing factor (CRF) in the hypothalamus, and also reduce cortisol in the adrenal gland and plasma in depressed rats [28]. The HPA axis has many functions including regulation of appetite, sleep, sexual desires and adaptation to stress; dysfunction of the HPA axis is thought to be primarily responsible for psychological/behavioral symptoms (pain sensitivity, depression, fatigue) [29].

Second, it is generally agreed that acupuncture and EA can attenuate cognitive deficits by means of inhibiting the neuronic peroxidatic reaction with the hippocampal tissue and attenuating the inflammation in the central and peripheral nervous systems [30], and one of the major pathways is the cholinergic anti-inflammatory pathway (CAP), which depends on the $\alpha 7$ subunit of the acetylcholine receptor (AChR) on macrophages and other cells [31]. It was demonstrated that EA treatment could activate the CAP by stimulating the vagus nerve for which the major neurotransmitter is acetylcholine. Previous reports showed that vagus nerve stimulation could inhibit the production of pro-inflammatory cytokines [32]. We speculated that TEAS might be an effective method to improve postoperative cognitive function.

Third, an increasing number of clinical trials indicate that acupuncture and EA may be effective in reducing perioperative analgesic requirements and postoperative pain [21]. Studies demonstrate that acupuncture and EA could produce endogenous opioid peptides and stimulate endogenous descending inhibitory pathways. Analysis of cerebrospinal fluid from patients receiving acupuncture treatment reveals elevated levels of serotonin, endorphins and enkephalin [33]. Previous animal and human studies indicate that low-frequency $(2 \mathrm{~Hz})$ and high-frequency $(100 \mathrm{~Hz})$ EA induces induces differences in the release of a variety of opioid peptides. Studies have shown that TEAS at $2 \mathrm{~Hz}$ induces release of enkephlins and endorphine, TEAS at $100 \mathrm{~Hz}$ induces release of dynorphine [34-36] and only TEAS at $2 / 100 \mathrm{~Hz}$ induces release of all opioid peptides, resulting in their synergistic interaction [37]. This may explain our observation that TEAS could ameliorate postoperative pain and reduce analgesic rescue therapy in patients undergoing gynecological laparoscopic surgery.

Fourth, stimulation of the acupoint Neiguan (PC6) on the pericardium meridian has been used for centuries in China to treat morning and travel sickness and has been reported to be effective in preventing postoperative nausea and vomiting [38, 39]. The anti-PONV effects of acupuncture may be attributed to changes in the activity of neurochemicals, including endorphins, serotonin and norepinephrine in the central nervous system, which desensitize the "vomiting center" in the brain, ultimately strengthen the intrinsic anti-vomiting pathway; once the vomiting center in the brain is sensitized, it is difficult to desensitize it [40]. We assume that TEAS initiates release of more neurochemicals at an early stage and immediately desensitizes the vomiting center.

Table 3 Incidence of nausea and vomiting, postoperative pain medications and antiemetics

\begin{tabular}{llll}
\hline Group & Cases of remedial analgesia & Cases of remedial antiemetic & PONV (\%) \\
\hline Con & $24(80 \%)$ & $16(53.3 \%)$ & $17(56.7 \%)$ \\
TEAS & $13^{*}(43.3 \%)$ & $7^{*}(23.3 \%)$ & $7^{*}(23.3 \%)$ \\
$P$ value & $P<0.01$ & $P<0.02$ & $P<0.01$ \\
\hline
\end{tabular}

Nausea was defined as a subjective unpleasant sensation associated with awareness of the urge to vomit; vomiting was defined as the forceful expulsion of gastric contents from the mouth brought about by the powerful sustained contraction of abdominal muscles PONV Postoperative nausea and vomiting, Con control group, TEAS transcutaneous electrical acupoint stimulation ${ }^{*} P<0.05$ vs. control group 
However, there were some limitations of this study. First, the sample size of 60 was small and comprised patients classified as ASA I and ASA II; a more diverse sample may have provided greater power to detect the effect of TEAS on the quality of early recovery in patients undergoing gynecological laparoscopic surgery. Second, the short study duration only permitted collection of limited data, which led to an underpowered trial. Third, due to economic restrictions we did not plan external monitoring and auditing. Thus, we cannot prove that our data really exits or that it is free from errors. However, for the sake of transparency, we state this limitation and we publish our results to the best of our understanding. Of course, before considering those results as definitive, they should be reproduced in a new trial with the highest standards for planning, monitoring, auditing and reporting.

\section{Conclusion}

In conclusion, we found that TEAS significantly promoted the quality of early recovery, improved cognitive function, ameliorated postoperative pain and reduced the incidence of nausea and vomiting within the first 24 of surgery. In future studies, different acupoints could be used for different types of surgery on other parts of the body. We also plan to investigate the underlying mechanisms of TEAS-mediated therapy, ultimately to broaden the clinical appeal of the technique.

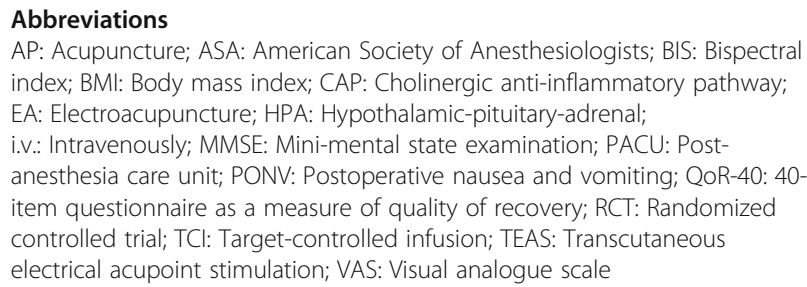

Abbreviations

AP: Acupuncture; ASA: American Society of Anesthesiologists; BIS: Bispectral index; BMI: Body mass index; CAP: Cholinergic anti-inflammatory pathway; EA: Electroacupuncture; HPA: Hypothalamic-pituitary-adrenal; i.v.: Intravenously; MMSE: Mini-mental state examination; PACU: Postanesthesia care unit; PONV: Postoperative nausea and vomiting; QoR-40: 40item questionnaire as a measure of quality of recovery; RCT: Randomized controlled trial; TCl: Target-controlled infusion; TEAS: Transcutaneous electrical acupoint stimulation; VAS: Visual analogue scale

\section{Authors' contributions}

FZ conceived and designed the experiments and contributed reagents, materials and analysis tools; XY performed the experiments, analyzed the data and wrote the manuscript; BC performed the experiments and contributed reagents, materials and analysis tools. All authors read and approved the final manuscript.

\section{Funding}

This work was supported by National natural science foundation of China, grant number 81660218; Guizhou Provincial Science and Technology Foundation, grant number Qiankehejichu [2016]1092, Qiankehejichu [2017]1108 and Qiankehejichu [2017]1107; Guizhou Provincial High-level creative talents cultivation plan: Thousand plan, grant number GZSYQCC [2016]001; Department of Science and Technology of Guizhou Province of China, grant number [2018]5764-07.

\section{Availability of data and materials}

Supporting data are available at clinicaltrials.gov (NCT02619578).

\section{Ethics approval and consent to participate}

The protocol was approved by the Ethics Committee of Guizhou Provincial People's Hospital, number 2013106. This study had all necessary consent approved by the Ethics Committee of Guizhou Provincial People's Hospital for any patients who will be involved in the study, including an informed consent form that patients will need to sign to participate in this study. The identity of the patients will not be disclosed. Individual patient data will be protected in order to protect patients' confidentiality.

\section{Consent for publication}

Not applicable.

\section{Competing interests}

The authors declare that they have no competing interests.

Received: 18 December 2016 Accepted: 6 November 2019

Published online: 08 January 2020

\section{References}

1. Chapron C, Fauconnier A, Goffinet F, Bréart G, Dubuisson JB. Laparoscopic surgery is not inherently dangerous for patients presenting with benign gynaecologic pathology. Results of a meta-analysis. Hum Reprod. 2002;17: 1334-42.

2. Nieboer TE, Johnson N, Lethaby A, Tavender E, Curr E, Garry R, et al. Surgical approach to hysterectomy for benign gynaecological disease. Cochrane Database Syst Rev. 2009;(3):CD003677.

3. Medeiros LR, Stein AT, Fachel J, Garry R, Furness S. Laparoscopy versus laparotomy for benign ovarian tumor: a systematic review and metaanalysis. Int J Gynecol Cancer. 2008;18:387-99.

4. Zhou C, Gong Z, Hu S. Influence of carbon dioxide pneumoperitoneum in laparoscopic cholecystectomy on respiration and circulation of middle-aged and elderly patients. China J Endosc. 2005;11:657-9.

5. Henny CP, Hofland J. Laparoscopic surgery: pitfalls due to anesthesia, positioning and pneumoperitoneum. Surg Endosc. 2005;19:1163-71.

6. Wang $\mathrm{H}$, Xie $\mathrm{Y}$, Zhang $\mathrm{Q}$, et al. Transcutaneous electric acupoint stimulation reduces intra-operative remifentanil consumption and alleviates postoperative side-effects in patients undergoing sinusotomy: a prospective, randomized, placebo-controlled trial. Br J Anaesth. 2014;112:1075-82.

7. Wetzel B, Pavlovic D, Kuse $R$, et al. The effect of auricular acupuncture on fentanyl requirement during hip arthroplasty: a randomized controlled trial. Clin J Pain. 2011:27:262-7.

8. Arnberger M, Stadelmann $\mathrm{K}$, Alischer $\mathrm{P}$, et al. Monitoring of neuromuscular blockade at the P6 acupuncture point reduces the incidence of postoperative nausea and vomiting. Anesthesiology. 2007;107:903-8.

9. Ni X, Xie Y, Wang Q, et al. Cardioprotective effect of transcutaneous electric acupoint stimulation in the pediatric cardiac patients: a randomized controlled clinical trial. Paediatr Anaesth. 2012;22:805-11.

10. Myles PS, Weitkamp B, Jones $\mathrm{K}$, et al. Validity and reliability of a postoperative quality of recovery score: the QoR-40. Br J Anaesth. 2000;84: $11-5$.

11. Myles PS, Reeves MD, Anderson $\mathrm{H}$, et al. Measurement of quality of recovery in 5672 patients after anaesthesia and surgery. Anaesth Intensive Care. 2000; 28:276-80.

12. De Oliveira GS Jr, Ahmad S, Fitzgerald PC, et al. Dose ranging study on the effect of preoperative dexamethasone on postoperative quality of recovery and opioid consumption after ambulatory gynaecological surgery. $\mathrm{Br}$ J Anaesth. 2011;107:362-71.

13. Myles PS, Williams DL, Hendrata M, et al. Patient satisfaction after anaesthesia and surgery: results of a prospective survey of 10,811 patients. Br J Anaesth. 2000;84:6-10

14. Wu CL, Richman JM. Postoperative pain and quality of recovery. Curr Opin Anaesthesiol. 2004;17:455-60.

15. Dorans N, Kulick E. Differential item functioning on the mini-mental state examination: an application of the Mantel-Haenszel and standardization procedures. Med Care. 2006;44(Suppl 3):S107-14

16. Kindler $\mathrm{CH}$, Harms $\mathrm{C}$, Amsler $\mathrm{F}$, et al. The visual analog scale allows effective measurement of preoperative anxiety and detection of patients' anesthetic concerns. Anesth Analg. 2000;90:706-12.

17. Gallagher EJ, Bijur PE, Latimer C, et al. Reliability and validity of a visual analog scale for acute abdominal pain in the ED. Am J Emerg Med. 2002;20: 287-90.

18. Andersson LE, Baath M, Thorne A, Aspelin P, Odeberg-Wernerman S. Effect of carbon dioxide pneumoperitoneum on development of atelectasis during anesthesia, examined by spiral computed tomography. Anesthesiology. 2005;102:293-9. 
19. Wu CL, Berenholtz SM, Pronovost PJ, Fleisher LA. Systematic review and analysis of post discharge symptoms after outpatient surgery. Anestheslology. 2002;96:994-1003.

20. Kotani N, Hashimoto H, Sato Y, et al. Preoperative intradermal acupuncture reduces postoperative pain, nausea and vomiting, analgesic requirement, and sympathoadrenal responses. Anesthesiology. 2001;95:349-5.

21. Sun Y, Gan TJ, Dubose JW, Habib AS. Acupuncture and related techniques for postoperative pain: a systematic review of randomized controlled trials. Br J Anaesth. 2008;101:151-60.

22. Shufang F. Electroacupuncture pretreatment ameliorates hypergravityinduced impairment of learning and memory and apoptosis of hippocampal neurons in rats. Neurosci Lett. 2010;478:150-5.

23. Kang JM. Acupuncture inhibits microglial activation and inflammatory events in the MPTP-induced mouse model. Brain Res. 2007;1131:211-9.

24. Liu L, Hoang-Gia T, Wu H, Lee MR, Gu L, Wang C, Yun BS, Wang Q, Ye S, Sung CK. Ginsenoside Rb1 improves spatial learning and memory by regulation of cell genesis in the hippocampal subregions of rats. Brain Res. 2011;1382:147-54

25. Zhang Z, Wang C, Li Q, Zhang M, Zhao H, Dong L, Wang G, Jin Y. Electroacupuncture at ST36 accelerates the recovery of gastrointestinal motility after colorectal surgery: a randomized controlled trial. Acupunct Med. 2014:32(3):223-6.

26. Lu F, Zhu HM, Xie JJ, et al. Effects of electroacupuncture on behavior, plasma COR and expression of PKA and PKC in hippocampus of depression model rat. Zhonghuo Zhen Jiu. 2008;28:214-8.

27. Chen HD, Jin LQ, Lou R. Effects of electroacupuncture on Baihui (GV20) for hypothalamic-pituitary-adrenal axis of chronic mild stress depression model rat. J Zhejiang Univ Tradit Chin Med. 2010;34:252-3.

28. Shi RX, Wu Q, Qin $L N$, et al. The effect of eletroacupuncture at "Baihui", "Yingtang" on weight and HPA axis of depression rats. J Clin Acupunct Moxib. 2007;23:50-3.

29. Dantzer R, O'Connor JC, Freund GG, Johnson RW, Kelley KW. From inflammation to sickness and depression: when the immune system subjugates the brain. Nat Rev Neurosci. 2008;9(1):46-56.

30. Yuan S, Zhang X, Bo Y, Li W, Zhang H, Jiang Q. The effects of electroacupuncture treatment on the postoperative cognitive function in aged rats with acute myocardial ischemia-reperfusion. Brain Res. 2014;17: 1593:19-29.

31. Tracey KJ. Physiology and immunology of the cholinergic anti-inflammatory pathway. J Clin Investig. 2007;117:289-96.

32. Das UN. Vagus nerve stimulation, depression and inflammation. Neuropsychopharmacology. 2007;32:2053-4.

33. Ulett GA, Han S, Han JS. Electroacupuncture: mechanisms and clinical application. Biol Psychiatry. 1998;44:129-38.

34. Han JS. Acupuncture: neuropeptide release produced by electrical stimulation of different frequencies. Trends Neurosci. 2003;26:17-22.

35. Han JS, Chen XH, Sun SL, Xu XJ, Yuan Y, Yan SC, Hao JX, Terenius L. Effect of low- and high-frequency TENS on Metenkephalin-Arg-Phe and dynorphin A immunoreactivity in human lumbar CSF. Pain. 1991;47:295-8.

36. Zhao SZ, Chung F, Hanna DB, Raymundo AL, Cheung RY, Chen C. Doseresponse relationship between opioid use and adverse effects after ambulatory surgery. J Pain Symptom Manag. 2004;28:35-46.

37. Han JS. Acupuncture and endorphins. Neurosci Lett. 2004;361:258-61.

38. Shyr MH, Hsu JC, Wu YW, Hui YL, Tan PC. P6 acupoint injection reduced postoperative nausea and vomiting. Anacsth Sinica. 1990;28:361-5.

39. Dundek JW, Cheskutt WN, Ghaly RG, Lynas AGA. Traditional Chinese acupuncture a potentially useful antiemetic? Br Med. 1986;293:583-4.

40. Wang XQ, Yu JL, Du ZY, Xu R, Jiang CC, Gao X. Electroacupoint stimulation for postoperative nausea and vomiting in patients undergoing supratentorial craniotomy. J Neurosurg Anesthesiol. 2010;22:128-31.

\section{Publisher's Note}

Springer Nature remains neutral with regard to jurisdictional claims in published maps and institutional affiliations.

Ready to submit your research? Choose BMC and benefit from:

- fast, convenient online submission

- thorough peer review by experienced researchers in your field

- rapid publication on acceptance

- support for research data, including large and complex data types

- gold Open Access which fosters wider collaboration and increased citations

- maximum visibility for your research: over $100 \mathrm{M}$ website views per year

At BMC, research is always in progress.

Learn more biomedcentral.com/submissions 